\title{
Ex vivo cytokine release reflects sensitivity to occupational endotoxin exposure
}

\author{
L.A.M. Smit*, D. Heederik*, G. Doekes*, E.J.M. Krop*, \\ G.T. Rijkers ${ }^{\#, \oplus}$ and I.M. Wouters*
}

ABSTRACT: Not everyone exposed to endotoxin develops respiratory symptoms, even at very high exposure levels. The aim was to investigate whether ex vivo lipopolysaccharide (LPS)induced cytokine release may be predictive of individual sensitivity to occupational endotoxin exposure.

In 412 agricultural workers, tumour necrosis factor (TNF)- $\alpha$, interleukin (IL)-1 $\beta$ and IL-10 release was measured in supernatants from LPS-stimulated whole blood, lung function was measured, and respiratory symptoms were assessed by questionnaire. For each cytokine, the population was dichotomised into low and high responders according to median cytokine concentrations. Endotoxin exposure levels were determined based on 249 personal exposure measurements.

High IL-10 responders had a higher prevalence of airway symptoms than low IL-10 responders (odds ratios between 2.03 and $5.10 ; p<0.05$ ). TNF- $\alpha$ response was positively, but not significantly, associated with symptoms, whereas no relationship was found between IL-1ß response and symptoms. For all three cytokines, subjects with above-median responses showed significant positive dose-response relationships between endotoxin exposure and asthma symptoms, and significant associations between endotoxin exposure and a lower forced expiratory volume in $1 \mathrm{~s}$ $(p<0.05)$. In contrast, exposure-response relationships were weak and statistically nonsignificant for low responders.

The ex vivo inflammatory response to LPS reflects, to a large extent, whether individuals are susceptible to adverse respiratory effects induced by high occupational endotoxin exposure.

KEYWORDS: Asthma, epidemiology, farming, lung function, occupational exposure, whole blood assay

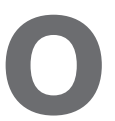
ccupational exposure to airborne endotoxins, cell wall components of Gramnegative bacteria, is associated with respiratory health effects in a dose-dependent manner. Epidemiological studies in occupationally exposed populations like farmers and cotton textile workers have shown exposure-response relationships between endotoxin exposure and acute lung function changes, accelerated chronic lung function decline and nonallergic asthma [1-8]. Airway inflammatory responses to inhaled endotoxin are characterised by increased levels of infiltrating neutrophils and production of proinflammatory cytokines, as shown by nasal lavage, induced sputum and bronchoalveolar lavage studies $[9,10]$.

Significant exposure-response relationships were recently reported between occupational endotoxin exposure and asthma-like symptoms in a large adult population of farmers and agricultural processing workers [1]. The majority of exposed subjects did not, however, report any respiratory symptoms, even at very high endotoxin exposure levels. Individual differences in sensitivity to inhaled endotoxin are likely to play a role and were already described more than two decades ago [11-13]. CASTELLAN et al. [11] screened naïve, healthy volunteers with a normal baseline forced expiratory volume in $1 \mathrm{~s}$ (FEV1) for inclusion in short-term endotoxin inhalation experiments. After precautionary exclusion of those with a decrease in FEV $1>30 \%$ on their first exposure, the authors found that endotoxin exposure elicited a measurable response in approximately one third of the remaining subjects, whereas others did not respond at all [11]. The precise biological mechanism that explains that some individuals appear to be more sensitive to inhaled endotoxin than others is not known, although multiple genetic factors arguably play an important role. For instance, polymorphisms in genes of the innate immunity pathway, such as Toll-like receptor 4 and CD14, have been
AFFILIATIONS

*Institute for Risk Assessment

Sciences, Division Environmental Epidemiology, Utrecht University, ${ }^{\#}$ Dept of Paediatrics, University Medical Center Utrecht, Utrecht, and "St Antonius Hospital, Dept of Medical Microbiology and Immunology, Nieuwegein, The Netherlands.

\section{CORRESPONDENCE}

L.A.M. Smit

Institute for Risk Assessment Sciences

Division Environmental Epidemiology P.0. Box 80178 3508 TD Utrecht The Netherlands E-mail: L.A.Smit@uu.nl

\section{Received:} Oct 272008 Accepted after revision: March 302009 First published online: April 082009 
associated with endotoxin responsiveness in experimental, occupational and domestic settings [14-17]. It has also been shown that responsiveness to inhaled lipopolysaccharide (LPS) is correlated with the ability of inflammatory cells to produce cytokines in response to LPS stimulation [13]. We therefore tested the hypothesis that heterogeneity in respiratory responses to occupational endotoxin exposure may be explained by inter-individual variation in cytokine responsiveness of LPS-stimulated inflammatory cells. We measured cytokine responses to purified endotoxin (LPS) in an ex vivo whole blood assay (WBA). Production of inflammatory cytokines tumour necrosis factor (TNF)- $\alpha$ and interleukin (IL)-1 $\beta$ were measured, as these cytokines are known to play a key role in endotoxin-induced inflammatory responses. In addition, the regulatory cytokine IL-10 was measured. The specific objectives of the present study were to investigate associations between LPS-induced cytokine release and respiratory health outcomes in a population with medium and high exposure to airborne endotoxin and, more specifically, to study whether the thus defined cytokine responsiveness modifies associations between endotoxin exposure and respiratory health parameters.

\section{METHODS}

\section{Study population}

The study population consisted of 434 Dutch agricultural processing workers and farmers sampled from a previously described survey [1, 18]. An overview of the recruitment is presented in figure 1. A total of 342 agricultural processing workers ( $91 \%$ of the 376 eligible) consented to blood sampling. A total of 340 farmers (65\% of the 525 eligible) indicated that they would not object to being contacted for a medical examination. For logistical reasons, however, only approximately 100 farmers could be included. A random sample of 122 farmers was approached by telephone, and 92 (75\%) participated.
During a visit to the worksite at the start of the workday, venous blood samples were taken for ex vivo cytokine induction in a WBA, for monocyte counts that were determined using an automated haematology analyser (Cell-Dyn Sapphire; Abbott Diagnostics, Abbott Park, IL, USA), and for serology. Lung function was assessed according to standard guidelines [19]. A standardised questionnaire with items of the European Community Respiratory Health Survey questionnaire was used to assess asthma-like symptoms [1, 20]. Atopy was defined as serum immunoglobulin $\mathrm{E}$ to one or more common allergens [18].

In total, data from 412 out of 434 participating subjects were used (323 agricultural processing workers and 89 farmers), because WBA could not be performed with blood from 15 subjects and we excluded four subjects aged $<18$ yrs or $>65$ yrs and three subjects with incomplete data. Farmers who participated in the medical examination did not differ significantly from farmers in the original sample with respect to endotoxin exposure, personal characteristics and the prevalence of reported wheeze ( $\mathrm{p}>0.05$; unpaired t-test or Chi-squared test). Nevertheless, selection bias in farmers was a potential concern and, therefore, sensitivity analyses were performed by repeating all statistical analyses in the subpopulation of agricultural processing workers only. The study protocol was approved by the institutional ethics committee (University Medical Center Utrecht, the Netherlands) and all participants gave written informed consent.

\section{Endotoxin exposure}

Endotoxin sampling, quantification and modelling was as described previously [1]. Briefly, 249 personal full-shift inhalable dust samples were collected on glass fibre filters using portable pumps and GSP (Gesamt Staub Probenehmer) sampling heads. Filters were extracted and supernatants were analysed by the quantitative kinetic chromogenic limulus

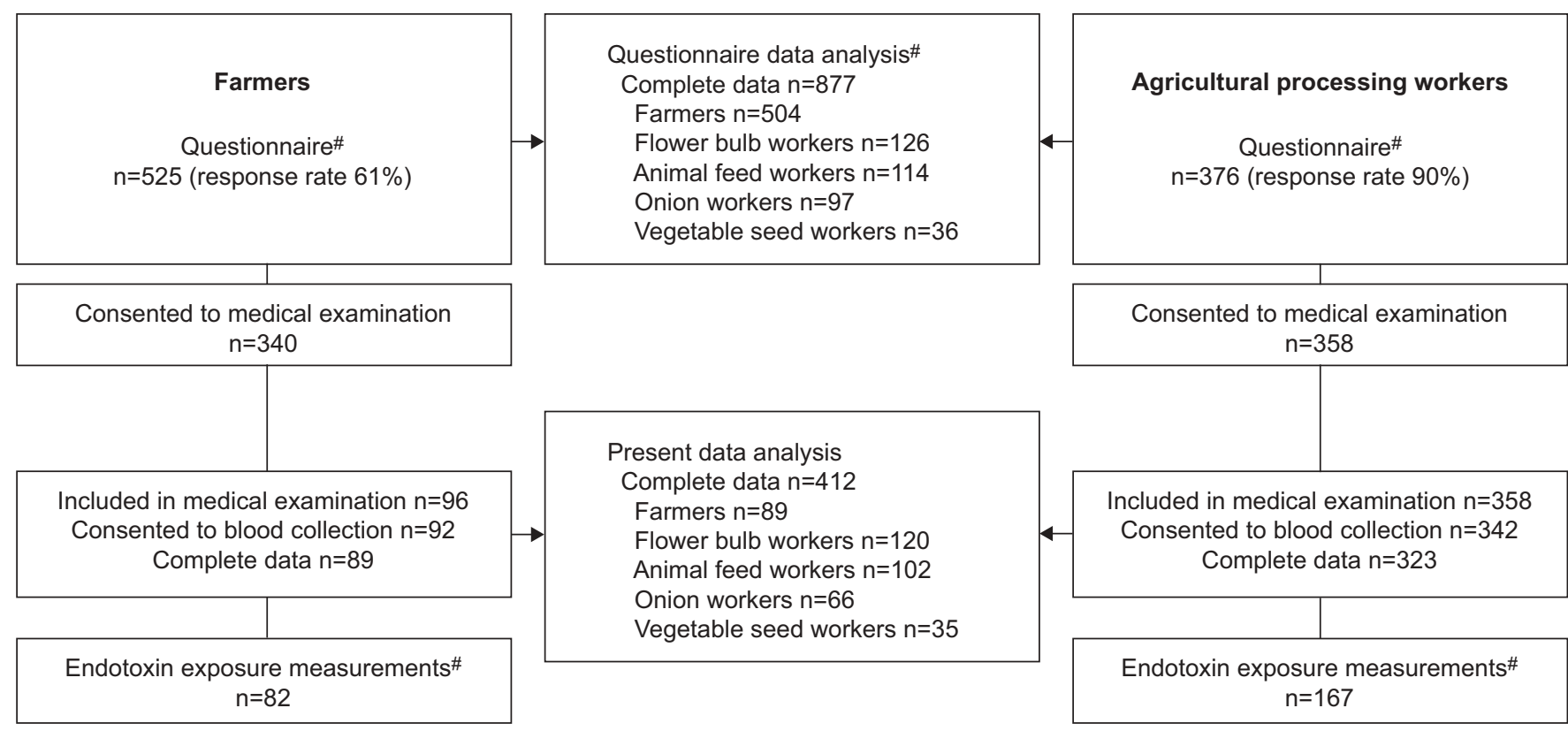

FIGURE 1. Flow diagram representing study population recruitment. * : according to reference [1]. 
amoebocyte lysate (LAL) assay. Airborne endotoxin exposure levels were assigned to each participant using a model that predicted endotoxin exposure by work environment and job title. Endotoxin exposure levels ranged over three orders of magnitude $\left(\sim 10-10,000\right.$ endotoxin units $\left.(\mathrm{EU}) \cdot \mathrm{m}^{-3}\right)$, and the geometric mean $(\mathrm{GM})$ in the present study $\left(319 \mathrm{EU} \cdot \mathrm{m}^{-3}\right)$ was similar to the GM of the original sample $\left(265 \mathrm{EU} \cdot \mathrm{m}^{-3}\right)$.

\section{Whole blood assay}

The procedure for LPS stimulation in a WBA was based on preliminary optimisation studies, in which heparin- and EDTA-anticoagulated blood of between two and 12 healthy donors was incubated with or without LPS or other stimuli in various concentrations, and cell supernatants were harvested after 1 or 2 days of culture [21].

In the present study, heparinised blood samples were kept on ice until use in the WBA. The average time between blood collection and start of the cell culture was $4.1 \pm 1.4 \mathrm{~h}$. Whole blood samples $(80-\mu \mathrm{L}$ aliquots) were transferred into sterile round-bottom 96-well microtitre plates (Greiner, Alphen a/d Rijn, the Netherlands) and diluted with an equal volume of RPMI 1640 medium supplemented with $100 \mathrm{U} \cdot \mathrm{mL}^{-1}$ penicillin and $100 \mu \mathrm{g} \cdot \mathrm{mL}^{-1}$ streptomycin (Gibco, Breda, the Netherlands). LPS (Fluka, Buchs, Switzerland) was added to the culture medium to a final concentration of $1 \mathrm{ng} \cdot \mathrm{mL}^{-1}\left(\sim 91 \mathrm{EU} \cdot \mathrm{mL}^{-1}\right.$ in the LAL assay). For each subject, negative controls were included by using medium without LPS. After culture for $18 \mathrm{~h}$ at $37^{\circ} \mathrm{C}, 5 \% \mathrm{CO}_{2}$ and $96 \%$ relative humidity, the plates were centrifuged (15 min at $100 \times g)$ and supernatants were stored at $-20^{\circ} \mathrm{C}$. TNF- $\alpha$, IL- $1 \beta$ and IL-10 were measured in supernatants using a Bio-plex assay [22]. The lower detection limits were $2.2 \mathrm{pg} \cdot \mathrm{mL}^{-1}, 20.8 \mathrm{pg} \cdot \mathrm{mL}^{-1}$ and $2.3 \mathrm{pg} \cdot \mathrm{mL}^{-1}$ for TNF- $\alpha, \mathrm{IL}-1 \beta$ and IL-10 respectively. On the basis of the preliminary results in 12 subjects [21], it was decided not to measure IL-6 and IL-8, as intra-individual variation exceeded inter-individual variation for these cytokines.

LPS-induced cytokine production was calculated by subtracting cytokine concentrations in the negative control wells from corresponding LPS-stimulated samples. Cytokine concentrations were inversely related to the time that elapsed between venapuncture and start of the cell culture: TNF- $\alpha$, IL- $1 \beta$ and IL10 production decreased by 32,12 and $8 \%$ per hour, respectively. Therefore, cytokine concentrations were adjusted to $t=0 \mathrm{~h}$ by applying a correction factor. For each cytokine, the population was dichotomised into low and high responders according to median cytokine concentrations for each cytokine separately. Distribution of high and low cytokine responses over individuals was, however, hardly influenced by the correction for time-untilincubation. Categorisations on the basis of adjusted or unadjusted levels were $\sim 90 \%$ concordant.

\section{Data analysis}

Results from 412 individuals with complete data were available. Associations between cytokine production and respiratory symptoms were assessed by logistic regression analysis. Odds ratios (ORs) for associations between endotoxin exposure and respiratory symptoms were estimated in strata of low and high cytokine responders. Associations between lntransformed endotoxin exposure levels and respiratory symptoms were presented as ORs and 95\% confidence interval (CI) for an interquartile range (IQR) increase of endotoxin exposure [1]. The shape of the exposure-response relationships was further analysed by means of generalised additive modelling (smoothing; SAS Proc GAM; SAS Institute, Cary, NC, USA), using Akaike's information criterion to select the degree of smoothing [23]. Associations between endotoxin exposure and FEV1 in low and high cytokine responders were analysed by linear regression analysis in the 363 male subjects of the study population. In the main data analysis, cytokine levels were $a$ priori dichotomised by the median to keep all 412 subjects in the analyses. Additional sensitivity analyses were performed by using the upper and lower tertiles, by treating cytokine production as a continuous variable, and by comparing subjects with a low or high response for all three cytokines.

\section{RESULTS}

\section{Determinants of cytokine response}

Figure 2 shows LPS-induced cytokine levels. High variability in cytokine release between participants was observed (two to three orders of magnitude), in particular for TNF- $\alpha$. Production of the three cytokines was significantly correlated, with pairwise correlations between ln-transformed cytokine levels of 0.57 for IL- $1 \beta$ and IL-10, 0.63 for IL- $1 \beta$ and TNF- $\alpha$, and 0.61 for IL-10 and TNF- $\alpha$ (Pearson correlation coefficients, $\mathrm{p}<0.001)$. Females $(\mathrm{n}=49)$ had a significantly lower LPSinduced cytokine production than males, in particular for IL-1 $\beta$ (factor $0.78 ; \mathrm{p}=0.04$ ) and TNF- $\alpha$ (factor $0.56 ; \mathrm{p}=0.001$ ). The sex difference in cytokine production was independent of age or endotoxin exposure. Dichotomisation into low and high responders was therefore performed separately for males and females. Furthermore, we calculated cytokine levels adjusted for the number of blood monocytes, and for all three cytokines the concentrations per $1 \times 10^{6}$ monocytes correlated strongly with the nonadjusted cytokine levels per millilitre (Pearson's $r=0.93$ to $r=0.96$; ln-transformed levels). When we dichotomised the population into low and high responders on the basis of monocyte-adjusted cytokine levels, we found excellent concordance with classifications on the basis of nonadjusted levels (85-90\%).

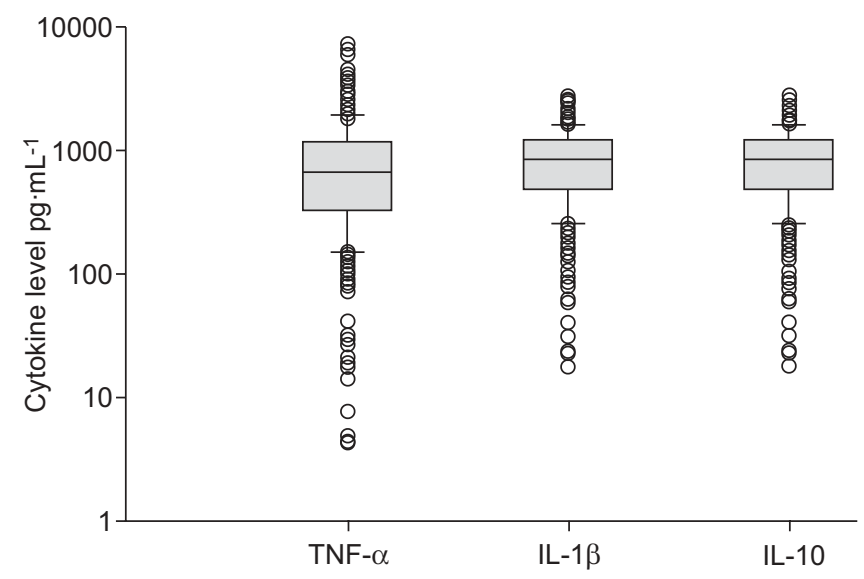

FIGURE 2. Box plots representing cytokine production in response to $1 \mathrm{ng} \cdot \mathrm{mL}^{-1}$ lipopolysaccharide in all study participants $(n=412)$. Boxes delineate the 25th and 75th percentiles with a horizontal line at the median, and whiskers depict the 10th and 90th percentiles. $\bigcirc$ : individual outliers. TNF: tumour necrosis factor; IL: interleukin. 
TABLE 1 Determinants of cytokine response

\begin{tabular}{|c|c|c|c|c|c|c|}
\hline & Low & High & Low & High & Low & High \\
\hline Age yrs & $42.6 \pm 10.2$ & $41.6 \pm 10.6$ & $42.9 \pm 9.7$ & $41.2 \pm 11.0$ & $43.9 \pm 9.5$ & $40.3 \pm 10.9^{*}$ \\
\hline \multicolumn{7}{|l|}{ Smoking habits } \\
\hline Never & 42.0 & 38.8 & 41.0 & 39.8 & 43.4 & 37.4 \\
\hline Farm childhood & 47.8 & 44.2 & 47.8 & 44.2 & 54.2 & $37.9^{\star}$ \\
\hline Atopy & 20.0 & 18.5 & 21.5 & 17.0 & 16.1 & 22.3 \\
\hline Blood monocytes $\times 10^{9} \cdot \mathrm{L}^{-1}$ & $0.53(1.4)$ & $0.55(1.4)$ & $0.51(1.3)$ & $0.57(1.4)^{*}$ & $0.52(1.4)$ & $0.56(1.3)^{*}$ \\
\hline Endotoxin exposure $\mathrm{EU} \cdot \mathrm{m}^{-3}$ & $276(4.9)$ & $307(5.5)$ & $238(5.1)$ & $356(5.2)^{\star}$ & $247(5.2)$ & $343(5.2)^{*}$ \\
\hline
\end{tabular}

Data are presented as mean $\pm S D, \%$ or geometric mean (geometric SD). The population was dichotomised into low $(n=206)$ and high $(n=206)$ responders according to median cytokine concentrations for each cytokine separately. TNF: tumour necrosis factor; IL: interleukin; EU: endotoxin units. *: p<0.05, unpaired t-test or Chi-squared test.

Table 1 shows the distribution of potential determinants over high and low TNF- $\alpha$, IL-1 $\beta$ and IL-10 responders. There was no association with smoking habits or atopy for any of the cytokines. Blood monocyte counts were higher in high IL-1 $\beta$ and IL-10 responders than in low responders $\left(0.57 \times 10^{9}\right.$ and $0.56 \times 10^{9}$ versus $0.51 \times 10^{9}$ and $0.52 \times 10^{9} \mathrm{~L}^{-1}$, respectively; $\mathrm{p}<0.05)$. High IL-10 responders were on average 3.5 yrs younger and had less often grown up on a farm than low IL10 responders. These relationships could also be confirmed among nonfarmers $(\mathrm{p}<0.05)$. Higher age and a farm childhood were independent determinants of a lower IL-10 response in a multiple logistic regression model with ORs of 0.97 (95\% CI 0.95-0.99) and 0.57 (95\% CI 0.38-0.85), respectively. Monocyte levels were also independently associated with IL-1 $\beta$ and IL10. Analyses with cytokine concentrations as continuous variables or upper and lower tertiles yielded very similar results.

A weak but statistically significant positive association between occupational endotoxin exposure and high IL-1 $\beta$ and IL-10 response was found. Although endotoxin exposure was associated with cytokine levels, the difference in average exposure levels between high and low responders was small and a strongly overlapping range of exposure levels was found in each subgroup. ORs for an IQR increase in endotoxin exposure were 1.25 (95\% CI 1.05-1.49) for IL-1 $\beta$ and 1.20 (95\% CI 1.01-1.42) for IL-10. When cytokine production was treated as a continuous variable, a significant but low correlation was also found between endotoxin exposure and IL-1 $\beta$ (lntransformed values, Pearson's $r=0.11, p=0.02)$, but not between exposure and IL-10 or TNF- $\alpha(\mathrm{p}>0.2)$. Endotoxin exposure was also weakly correlated with blood monocyte levels $(r=0.12, p=0.02)$, and adjustment for monocytes slightly attenuated the associations between endotoxin exposure and IL-1 $\beta$ and IL-10.

\section{Associations between cytokine response and respiratory symptoms}

High IL-10 responders had wheeze symptoms more often than low IL-10 responders (table 2 ). TNF- $\alpha$ response was also positively, but nonsignificantly, associated with symptoms (ORs between 1.32 and 1.86), whereas no relationship between IL-1 $\beta$ response and symptoms was found. Cough symptoms (daily cough, cough up phlegm and nocturnal cough) were not associated with cytokine production (data not shown). No evidence of confounding or effect modification by variables

TABLE 2 Prevalence of respiratory symptoms in 206 low cytokine responders and in 206 high cytokine responders and association with cytokine responses above the median

\begin{tabular}{|c|c|c|c|c|c|c|c|c|c|}
\hline & \multicolumn{3}{|c|}{ TNF- $\alpha$ response } & \multicolumn{3}{|c|}{ IL-1 $\beta$ response } & \multicolumn{3}{|c|}{ IL-10 response } \\
\hline & Low & High & OR $(95 \% \mathrm{Cl})$ & Low & High & OR $(95 \% \mathrm{Cl})$ & Low & High & OR $(95 \% \mathrm{Cl})$ \\
\hline Wheezing with SOB & 7.3 & 9.7 & $1.32(0.65-2.67)$ & 9.3 & 7.8 & $0.77(0.38-1.57)$ & 4.9 & 12.1 & $2.58(1.18-5.66)^{*}$ \\
\hline Wheezing without a cold & 4.9 & 8.8 & $1.86(0.83-4.17)$ & 6.3 & 7.3 & $1.13(0.52-2.46)$ & 2.4 & 11.2 & $5.10(1.85-14.1)^{*}$ \\
\hline Awakened due to SOB & 2.4 & 4.4 & $1.77(0.58-5.41)$ & 3.4 & 3.4 & $0.95(0.32-2.79)$ & 1.5 & 5.3 & $3.51(0.93-13.2)$ \\
\hline
\end{tabular}

Data are presented as \%, unless otherwise stated. TNF: tumour necrosis factor; IL: interleukin; OR: odds ratio; Cl: confidence interval; SOB: shortness of breath. ORs were adjusted for sex, age, smoking habits and farm childhood. ${ }^{*}: p<0.05$ for associations between cytokine response and symptoms. 


\begin{tabular}{|c|c|c|c|c|c|c|}
\hline \multirow[t]{3}{*}{ TABLE 3} & $\begin{array}{l}\text { between occup } \\
\text { sytokine respor }\end{array}$ & $\begin{array}{l}\text { nal endotoxin ex } \\
\text { rs }\end{array}$ & sure and respir & ory symptoms i & 206 low cytokir & responders and \\
\hline & \multicolumn{2}{|c|}{ TNF- $\alpha$ response } & \multicolumn{2}{|c|}{ IL-1 $\beta$ response } & \multicolumn{2}{|c|}{ IL-10 response } \\
\hline & Low & High & Low & High & Low & High \\
\hline Wheezing & $1.02(0.66-1.56)$ & $1.82(1.24-2.67)^{* . \#}$ & $1.14(0.76-1.72)$ & $1.66(1.13-2.45)^{\star}$ & $1.12(0.70-1.78)$ & $1.54(1.08-2.20)^{*}$ \\
\hline Awakened due to SOB & $1.79(0.73-4.35)$ & $1.86(0.99-3.49)$ & $2.16(1.01-4.61)^{*}$ & $1.55(0.82-2.95)$ & $0.88(0.29-2.68)$ & $2.28(1.22-4.28)^{* .9}$ \\
\hline
\end{tabular}

such as age, farm childhood, smoking or atopy was found, and exclusion of farmers or females did not influence results. Endotoxin exposure was not a confounding factor for the association between cytokine response and respiratory outcomes, as was expected on the basis of the weak associations between endotoxin exposure and cytokine responses. Monocytes were not associated with symptoms ( $p>0.3$ for all symptoms), and adjustment for monocytes did not change the results. Similar patterns were observed when cytokine production was treated as a continuous variable, or when the lowest and highest tertiles of cytokine production were compared.

\section{Cytokine responses as effect modifiers}

A clear dose-response relationship was previously found between endotoxin exposure and the prevalence of respiratory symptoms [1], and we found similar results in the present study. In stratified analyses these dose-response relationships appeared to be largely restricted to the subjects with high cytokine responses (table 3). For all three cytokines, high responders showed significant positive relationships between occupational endotoxin exposure and wheeze, wheeze with shortness of breath and wheeze without a cold (ORs between 1.54 and 2.13). In contrast, weak and nonsignificant associations were found in low responders (ORs between 0.98 and 1.26). ORs for wheeze and wheeze with shortness of breath were significantly different between low and high TNF- $\alpha$ responders (formal test for interaction, $\mathrm{p}<0.05$ ). Exposure-response relationships between endotoxin exposure and cough symptoms were not modified by cytokine response (data not shown).

The shape of the relationships between endotoxin and wheeze seemed log-linear for both high and low TNF- $\alpha$ responders (fig. 3a). A nonlinear relationship was suggested for IL-1 $\beta$ and IL-10 high responders. A steep exposure-response relationship was observed, but only at very high endotoxin exposure levels $\left(>1,000 \mathrm{EU} \cdot \mathrm{m}^{-3}\right)$. Curves for low responders flattened at high exposures (fig. $3 \mathrm{~b}$ and $\mathrm{c}$; spline $\mathrm{p}<0.1$ ).

Results changed only marginally when female workers or farmers were excluded. In agricultural processing workers, ORs between 1.55 and 2.05 were found for wheeze-related symptoms in high cytokine responders, and between 0.78 and 1.29 for low responders.

FEV1 did not differ between low and high responders $(\mathrm{p}>0.2$; data not shown). However, in high TNF- $\alpha$, IL-1 $\beta$ and IL-10 responders, a significantly lower FEV1 was found at increasing
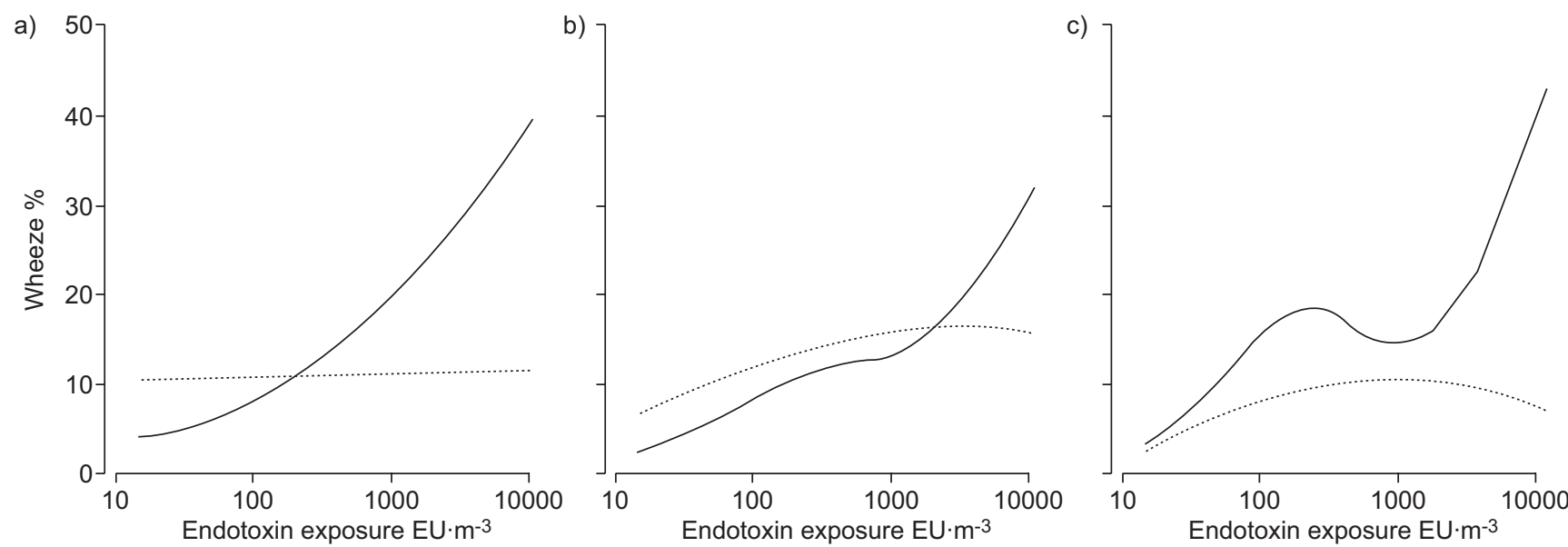

FIGURE 3. Smoothed plots representing dose-response relationships of endotoxin exposure and current wheeze in 206 high responders (—) and 206 low responders $(\cdots \cdots \ldots . .$.$) for a) tumour necrosis factor- \alpha$, b) interleukin (IL)-1 $\beta$ and c) IL-10. EU: endotoxin units. 


\begin{tabular}{|c|c|c|c|c|}
\hline \multirow[t]{3}{*}{ TABLE 4} & \multicolumn{4}{|c|}{$\begin{array}{l}\text { Association between occupational endotoxin } \\
\text { exposure and forced expiratory volume in } 1 \mathrm{~s} \\
(\mathrm{FEV} 1) \text { in } 363 \text { male subjects according to } \\
\text { cytokine response }\end{array}$} \\
\hline & \multicolumn{2}{|c|}{ Low responders ${ }^{\#}$} & \multicolumn{2}{|c|}{ High responders } \\
\hline & $\beta(95 \% \mathrm{Cl})$ & $p$-value & $\beta(95 \% \mathrm{Cl})$ & p-value \\
\hline TNF- $\alpha$ & $-30(-111-51)$ & 0.47 & $-80(-154--6)$ & 0.03 \\
\hline IL-1及 & $-18(-97-62)$ & 0.66 & $-80(-157--3)$ & 0.04 \\
\hline IL-10 & $-28(-103-47)$ & 0.46 & $-92(-170--14)$ & 0.02 \\
\hline \multicolumn{5}{|c|}{ 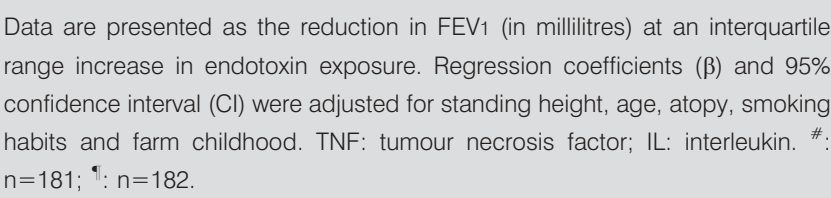 } \\
\hline
\end{tabular}

levels of endotoxin (80-92 mL lower for an IQR increase in endotoxin exposure; $\mathrm{p}<0.05)$, whereas in low responders a much smaller and nonsignificant difference in FEV1 (18-30 mL lower) was found at the same increase in exposure (table 4). Formal tests for interaction were, however, not significant for any of the cytokines. Similar results were obtained when analyses shown in tables 2-4 were repeated with low and high responders classified on the basis of monocyte-adjusted cytokine levels.

\section{Sensitivity analyses with combined cytokine response}

Since LPS-induced production of the three cytokines was highly correlated, we also combined responses into a single entity. A comparison of subjects with a low or high response to LPS for all three cytokines (low: $n=124,30.1 \%$; high: $n=123$, $29.9 \%)$, ignoring subjects with discordant responses $(n=165$, $40.1 \%$ ), showed that high cytokine production was positively associated with symptom prevalence, with ORs between 1.69 and 3.43 ( $p<0.05$ for wheeze without a cold). Effect modification of the exposure-response relationships between endotoxin exposure and symptoms or FEV1 was very similar to results shown in tables 3 and 4 . High responders had ORs between 1.68 and 2.07 for respiratory symptoms $(\mathrm{p}<0.05$ for wheeze, wheeze with SOB and wheeze without a cold) and a 172-mL lower FEV1 $(p=0.002)$ for an IQR increase in endotoxin exposure. In contrast, corresponding ORs for low responders were between 0.80 and 1.17 for symptoms $(p>0.05)$ and their FEV1 was on average only $27 \mathrm{~mL}$ lower when endotoxin exposure increased over an IQR. The interaction between cytokine response and endotoxin exposure was significant for the association with wheeze ( $p=0.03$ for interaction).

\section{DISCUSSION}

In the present study we found that susceptibility to adverse respiratory effects induced by high occupational endotoxin exposure was reflected by the inflammatory response to LPS, measured as ex vivo TNF- $\alpha$, IL- $1 \beta$ and IL-10 responses in a WBA. For high cytokine responders, exposure-response relationships between occupational endotoxin exposure and FEV1 or respiratory symptoms were relatively steep and statistically significant. In low cytokine responders, associations were weak and nonsignificant.

On theoretical grounds, dichotomisation of subjects into high and low responders according to median cytokine production would be in agreement with the finding that more than onethird of individuals appeared responsive to acute, experimental endotoxin inhalation [11]. Sensitivity analyses showed that results were robust when tertiles were used or when cytokine production was treated as a continuous variable. Moreover, the number of blood monocytes did not account for the differences in susceptibility between low and high responders. Heterogeneous results were observed for the three cytokines under study, in particular for associations between cytokine production and symptom prevalence. A possible biological meaning of this difference is, however, not directly available, and should be interpreted with caution. Conversely, categorisation based on all three cytokine responses together is expected to be less sensitive for measurement error of individual cytokines and, thus, this strengthened the finding that ex vivo LPS-induced cytokine response was associated with susceptibility to endotoxin-induced respiratory disorders. On the basis of our findings, it cannot be concluded whether the three different cytokine responses should be interpreted as three separate biologically meaningful responses. In vivo, the immunological roles of TNF- $\alpha$ and IL-1 $\beta$ as inflammatory mediators and IL-10 as an inhibitor of cytokines are quite distinct. However, we stimulated whole blood with LPS for $18 \mathrm{~h}$, resulting in stimulation of monocytes and not of IL-10-producing T-cells. Therefore, the cytokine responses could also be interpreted as a single correlated response of monocytes to LPS.

It has been shown that time between blood collection and start of incubation in the WBA is a critical variable for cytokine release [24, 25]. Although we made every effort to minimise this time period by optimising fieldwork and laboratory procedures, we could not start the in vitro incubation of all blood samples within $2 \mathrm{~h}$ of collection; therefore, all measured cytokine levels were adjusted for the time-until-incubation. In our study, variation in time-until-incubation was small and correction had only a minor impact on the categorisation of high and low responders.

We found lower TNF- $\alpha$ and IL-1 $\beta$ production from whole blood samples of females compared with males. A similar sex difference has been observed previously in a study among volunteers, and was attributed to the influence of sex hormones [26]. We therefore categorised males and females separately into high and low responders, and we also showed that results were very similar when we excluded females from analyses.

Prolonged exposure to high levels of endotoxin has been shown to lead to persistent airway symptoms and obstruction, probably as a result of chronic mucosal airway inflammation. Apparently, high occupational exposure may also have a systemic effect by increasing the number of circulating monocytes and enhancing the ability of blood monocytes to produce cytokines, as suggested by the weak but positive association between endotoxin exposure and IL-1 $\beta$ and IL-10 (table 1). Also, when cytokine responses were combined (all low versus all high), we found a positive association between 
endotoxin exposure and the cytokine response (OR 1.31, 95\% CI 1.04-1.66). In a previous study among grain workers, TNF- $\alpha$ release from unstimulated and stimulated peripheral blood monocytes ( $\left.3 \mathrm{ng} \cdot \mathrm{mL}^{-1} \mathrm{LPS}\right)$ increased significantly across the working week in exposed workers, and the increase in TNF- $\alpha$ levels was associated with grain dust exposure levels [27]. In our study, blood was collected from Monday to Wednesday at the start of the workday, and there was no association between day of the week and cytokine production $(p>0.3)$. A lower response in the WBA has been described in mice that had developed tolerance to LPS [28], but, in human studies including the present one, there is no evidence of development of tolerance at adult age to occupational endotoxin exposure, at least not at the level of cytokine responses [27].

Childhood exposure to microbial agents in a farm environment has been associated with lower LPS-induced IL-10 responses by BRAUN-FAHRLÄNDER et al. [29]. The study found an inverse association between endotoxin load in children's mattress dust and LPS-induced IL-10 [29]. Our finding that workers who had grown up on a farm had a lower IL-10 response (table 1) appears to be in accordance with that study. Whether our observation relates to a long-lasting downregulation of IL-10 responses as a result of childhood exposures to endotoxin and other farm-associated microbial agents, or to other factors correlated to a farm childhood, however, is not clear.

LPS-induced release of IL-6 and IL- 8 by alveolar macrophages and peripheral blood monocytes correlates with airflow obstruction in response to experimental LPS inhalation [13]. This suggests that cytokine release from inflammatory cells is predictive of in vivo susceptibility. A WBA is a simple alternative to stimulating isolated monocytes or alveolar macrophages, especially in large-scale population studies with complicated logistics. The WBA reproducibly measures an individual's cytokine responsiveness, with considerably larger differences in cytokine release between individuals than within individuals when repeated during a period of 1-6 weeks [24, $25,30]$. It is as yet unknown whether individual responses are reproducible over a longer period of time. Moreover, longitudinal studies are needed in order to assess the predictive value of cytokine responsiveness.

In conclusion, we have shown that TNF- $\alpha$, IL-1 $\beta$ and IL-10 production by ex vivo LPS-stimulated blood monocytes is associated with in vivo susceptibility to occupational endotoxin exposure-associated respiratory effects. Exposure-response relationships between endotoxin exposure and respiratory symptoms and FEV1 were relatively steep and statistically significant for high cytokine responders, whereas weak, nonsignificant associations were found for low cytokine responders.

\section{SUPPORT STATEMENT}

This study was funded by the Netherlands Asthma Foundation (Grant No. 3.2.03.70)

\section{STATEMENT OF INTEREST}

None declared.

\section{ACKNOWLEDGEMENTS}

M. Boeve, I. Oosting, J. Spithoven, M. Visser and S. de Wind are gratefully acknowledged for fieldwork and laboratory assistance (all at the Institute for Risk Assessment Sciences, Utrecht University, Utrecht, the Netherlands).

\section{REFERENCES}

1 Smit LAM, Heederik D, Doekes G, et al. Exposure-response analysis of allergy and respiratory symptoms in endotoxinexposed adults. Eur Respir J 2008; 31: 1241-1248.

2 Kennedy SM, Christiani DC, Eisen EA, et al. Cotton dust and endotoxin exposure-response relationships in cotton textile workers. Am Rev Respir Dis 1987; 135: 194-200.

3 Heederik D, Brouwer R, Biersteker K, et al. Relationship of airborne endotoxin and bacteria levels in pig farms with the lung function and respiratory symptoms of farmers. Int Arch Occup Environ Health 1991; 62: 595-601.

4 Smid T, Heederik D, Houba R, et al. Dust- and endotoxin-related respiratory effects in the animal feed industry. Am Rev Respir Dis 1992; 146: 1474-1479.

5 Sigsgaard T, Malmros P, Nersting L, et al. Respiratory disorders and atopy in Danish refuse workers. Am J Respir Crit Care Med 1994; 149: 1407-1412.

6 Vogelzang PF, van der Gulden JW, Folgering H, et al. Endotoxin exposure as a major determinant of lung function decline in pig farmers. Am J Respir Crit Care Med 1998; 157: 15-18.

7 Donham KJ, Cumro D, Reynolds SJ, et al. Dose-response relationships between occupational aerosol exposures and cross-shift declines of lung function in poultry workers: recommendations for exposure limits. J Occup Environ Med 2000; 42: 260-269.

8 Eduard W, Douwes J, Omenaas E, et al. Do farming exposures cause or prevent asthma? Results from a study of adult Norwegian farmers. Thorax 2004; 59: 381-386.

9 Thorn J. The inflammatory response in humans after inhalation of bacterial endotoxin: a review. Inflamm Res 2001; 50: 254-261.

10 Sigsgaard T, Bonefeld-Jorgensen EC, Hoffmann HJ, et al. Microbial cell wall agents as an occupational hazard. Toxicol Appl Pharmacol 2005; 207: 310-319.

11 Castellan RM, Olenchock SA, Kinsley KB, et al. Inhaled endotoxin and decreased spirometric values. An exposure-response relation for cotton dust. N Engl J Med 1987; 317: 605-610.

12 Michel O, Duchateau J, Sergysels R. Effect of inhaled endotoxin on bronchial reactivity in asthmatic and normal subjects. J Appl Physiol 1989; 66: 1059-1064.

13 Kline JN, Cowden JD, Hunninghake GW, et al. Variable airway responsiveness to inhaled lipopolysaccharide. Am J Respir Crit Care Med 1999; 160: 297-303.

14 Arbour NC, Lorenz E, Schutte BC, et al. TLR4 mutations are associated with endotoxin hyporesponsiveness in humans. Nat Genet 2000; 25: 187-191.

15 LeVan TD, Von Essen S, Romberger DJ, et al. Polymorphisms in the CD14 gene associated with pulmonary function in farmers. Am J Respir Crit Care Med 2005; 171: 773-779.

16 Smit LA, Bongers SI, Ruven HJ, et al. Atopy and new-onset asthma in young Danish farmers and CD14, TLR2, and TLR4 genetic polymorphisms: a nested case-control study. Clin Exp Allergy 2007; 37: 1602-1608.

17 Simpson A, John SL, Jury F, et al. Endotoxin exposure, CD14, and allergic disease: an interaction between genes and the environment. Am J Respir Crit Care Med 2006; 174: 386-392.

18 Smit LAM, Heederik D, Doekes G, et al. Exhaled nitric oxide in endotoxin-exposed adults: effect modification by smoking and atopy. Occup Environ Med 2009; 66: 251-255.

19 Quanjer PH, Tammeling GJ, Cotes JE, et al. Lung volumes and forced ventilatory flows. Report Working Party Standardization of Lung Function Tests, European Community for Steel and Coal. Official Statement of the European Respiratory Society. Eur Respir J 1993; 6: Suppl. 16, 5-40. 
20 Burney PG, Luczynska C, Chinn S, et al. The European Community Respiratory Health Survey. Eur Respir J 1994; 7: 954-960.

21 Smit LAM, Wouters IM, Heederik D, et al. Optimization of a whole blood assay for determining cytokine responses in occupational populations. Eur Respir J 2005; 26: Suppl. 49, 599s.

22 de Jager $W$, te Velthuis H, Prakken BJ, et al. Simultaneous detection of 15 human cytokines in a single sample of stimulated peripheral blood mononuclear cells. Clin Diagn Lab Immunol 2003; 10: 133-139.

23 Portengen L, Preller L, Tielen M, et al. Endotoxin exposure and atopic sensitization in adult pig farmers. J Allergy Clin Immunol 2005; 115: 797-802.

24 van der Linden MW, Huizinga TW, Stoeken DJ, et al. Determination of tumour necrosis factor- $\alpha$ and interleukin-10 production in a whole blood stimulation system: assessment of laboratory error and individual variation. J Immunol Methods 1998; 218: 63-71.
25 Wouters IM, Douwes J, Thorne PS, et al. Inter- and intraindividual variation of endotoxin- and $\beta(1,3)$-glucan-induced cytokine responses in a whole blood assay. Toxicol Ind Health 2002; 18: 15-27.

26 Aulock SV, Deininger S, Draing C, et al. Gender difference in cytokine secretion on immune stimulation with LPS and LTA. J Interferon Cytokine Res 2006; 26: 887-892.

27 Borm PJ, Schins RP, Derhaag TJ, et al. Cross-shift changes in blood inflammatory markers occur in the absence of airway obstruction in workers exposed to grain dust. Chest 1996; 109: 1078-1085.

28 Mueller-Anneling LJ, O'Neill ME, Thorne PS. Biomonitoring for assessment of organic dust-induced lung inflammation. Eur Respir J 2006; 27: 1096-1102.

29 Braun-Fahrländer C, Riedler J, Herz U, et al. Environmental exposure to endotoxin and its relation to asthma in school-age children. N Engl J Med 2002; 347: 869-877.

30 Yaqoob P, Newsholme EA, Calder PC. Comparison of cytokine production in cultures of whole human blood and purified mononuclear cells. Cytokine 1999; 11: 600-605. 